\title{
Differences in meat quality between boars and gilts
}

\author{
P. D. Warriss, S. N. Brown and G. R. Nute \\ Department of Meat Animal Science, University of Bristol, Langford, Bristol BS18 7DY
}

\section{Introduction}

The large majority of boars currently slaughtered in the United Kingdom are not castrated. Early work suggested that the eating quality of pork from boars was not different from that of gilts (Rhodes, 1972). Recent work (Meat and Livestock Commission, 1989) found that gilts produced meat which had better pork odour and less abnormal odour but there were no differences in flavour, juiciness or tenderness. The different conclusions could be due, in part at least to differences in the slaughter weight of the pigs since undesirable odours are associated with onset of sexual maturity in the male. Because of the importance of quality in maintaining sales of pork, aspects of raw meat quality and eating quality in a large sample of boars and gilts killed in various experiments at the University of Bristol slaughterhouse were examined. The average carcass weight was $70 \mathrm{~kg}$.

\section{Material and methods}

Information was selected from a matched set of 300 boars and 300 gilts. These had a range of genotypes but were of predominantly White breeding and representative of the majority of pigs killed in the United Kingdom at present. They were slaughtered using normal commercial practices. Hot carcass weight and $\mathrm{P}_{2}$ value were recorded. After overnight chilling a section of the loin joint $(\mathrm{m}$. longissimus dorsi) was removed for measurement of $\mathrm{pH}_{\mathrm{u}}$, colour using the CIELAB $L^{\star} a^{\star} b^{\star}$ system, drip loss during storage, and instrumental texture (shear force) as described by Warriss, Kestin, Rolph and Brown (1990). Eating quality was measured on deboned, griddled, $2.5 \mathrm{~cm}$ chops cooked to an internal temperature of $80^{\circ} \mathrm{C}$ and presented to a panel of 18 trained assessors. Samples were evaluated using eight-point category scales. Pork given higher ratings was more tender, more juicy, had stronger flavour/ odour, was darker and was overall more acceptable.

\section{Results and discussion}

Boars produced carcasses which were $1.5 \mathrm{~kg}$ lighter and had $1.1 \mathrm{~mm}$ less fat at the $\mathrm{P}_{2}$ position (Table 1 ). Ultimate $\mathrm{pH}$ was not affected by sex, neither were muscle lightness ( $\mathrm{L}^{\star}$ ) or colour saturation. Hue was significantly higher in meat from gilts which also lost slightly more drip during storage.

There was no difference in the texture of meat from boars or gilts, based on either instrumental measurements (Table 1) or taste panel assessments (Table 2), or in juiciness. However, the cooked lean from gilts was paler, had stronger pork flavour but less abnormal flavour, and was given a higher overall acceptability rating. Similarly, the cooked fat from gilts had stronger pork odour and less abnormal odour. Of the samples of lean from boars, $8.5 \%$ were given slightly to extremely strong abnormal flavour ratings compared with only $0.3 \%$

Table 1 Weight, fatness and lean meat quality in boars and gilts

\begin{tabular}{|c|c|c|c|}
\hline & Boars & Gilts & s.e.d. \\
\hline Live weight (kg) & $89 \cdot 0$ & 88.5 & 0.602 \\
\hline Hot carcass wt. $(\mathrm{kg})$ & 69.5 & $71 \cdot 0^{* * *}$ & $0 \cdot 207$ \\
\hline $\mathrm{P}_{2}(\mathrm{~mm})$ & $10 \cdot 0$ & $11 \cdot 1^{* * *}$ & 0.228 \\
\hline $\mathrm{pH}_{\mathrm{u}} \mathrm{LD}$ & 5.48 & $5 \cdot 46$ & 0.00821 \\
\hline Lightness (L ) & 54.5 & $54 \cdot 9$ & 0.228 \\
\hline Hue & $34 \cdot 0$ & $36 \cdot 1^{* *}$ & 0.686 \\
\hline Saturation & 6.5 & $6 \cdot 5$ & $0 \cdot 125$ \\
\hline Drip loss (\%) & $7 \cdot 2$ & $7 \cdot 9^{* *}$ & 0.247 \\
\hline Shear force $(\mathrm{kg})$ & $5 \cdot 13$ & $5 \cdot 19$ & $0 \cdot 106$ \\
\hline
\end{tabular}

All values, except live weight, corrected to a live weight of $88.7 \mathrm{~kg}$.

Table 2 Eating quality of meat from boars and gilts

\begin{tabular}{llll}
\hline \hline & Boars & Gilts & s.e.d. \\
\hline Fat & & & \\
$\quad$ pork odour & $3 \cdot 60$ & $3 \cdot 80^{* * *}$ & 0.0462 \\
$\quad$ abnormal odour & $3 \cdot 01$ & $2 \cdot 29^{* * *}$ & 0.0886 \\
Lean & & & \\
$\quad$ colour & 4.47 & $4 \cdot 32^{* * *}$ & 0.0410 \\
$\quad$ texture & 4.08 & $4 \cdot 17$ & 0.0649 \\
juiciness & 3.83 & $3 \cdot 79$ & 0.0423 \\
pork flavour & 3.71 & $3 \cdot 86^{* * *}$ & 0.0378 \\
abnormal flavour & 3.22 & $2 \cdot 76^{* * *}$ & $0 \cdot 0691$ \\
$\quad$ overall acceptability & 3.55 & $3.90^{* * *}$ & 0.0532 \\
\hline \hline
\end{tabular}

All values corrected to a live weight of $88.7 \mathrm{~kg}$. 


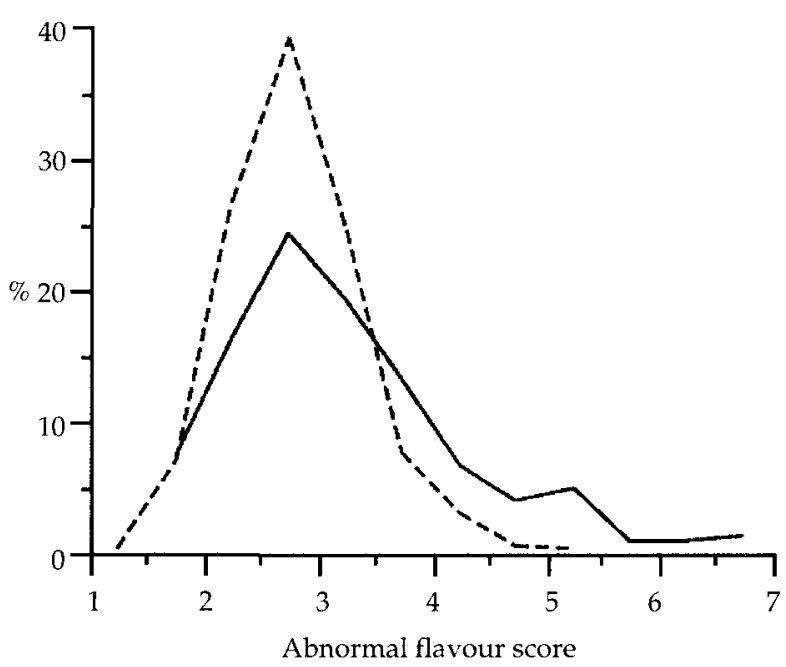

Figure 1 The distribution of abnormal flavour scores in lean pork from boars (-) and gilts (- -).

from gilts (Figure 1). Of the samples of fat, $11.7 \%$ from boars were given slightly to extremely strong odour ratings; the corresponding figure for gilts was $0.4 \%$ (Figure 2). The distributions of overall acceptability ratings are illustrated in Figure 3 . The size or significance of these differences between boars and gilts were not affected if $P_{2}$ value was included as a covariate to remove any influence of the difference in fatness between the sexes. However, overall $($ no. $=600)$, there were significant, but very weak correlations between $\mathrm{P}_{2}$ value and pork flavour

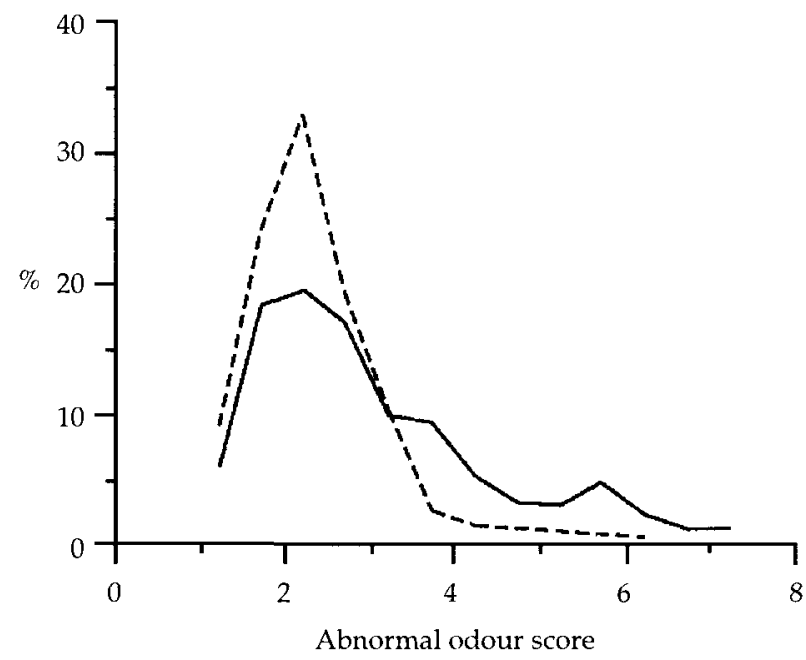

Figure 2 The distribution of abnormal odour scores in fat from boars (-) and gilts (-.).

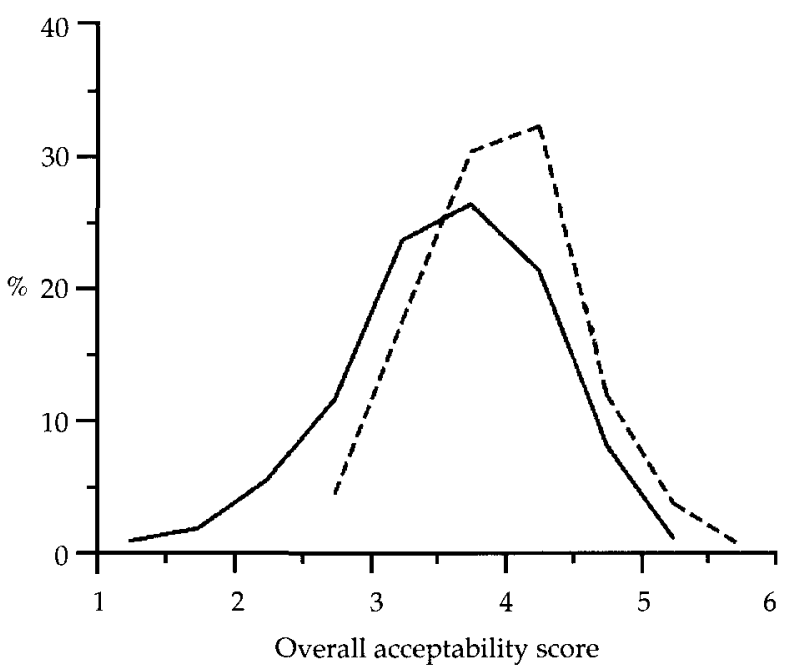

Figure 3 Overall acceptability scores for eating quality of pork from boars (-) and gilts (- - ).

of the lean $(r=0.17, P<0.01)$, juiciness $(r=0.10$, $P<0.05)$ and overall acceptability $(r=0.10, P<0.05)$.

Hot carcass weight ranged from less than $60 \mathrm{~kg}$ to nearly $90 \mathrm{~kg}$ but there was no evidence from overall correlations that abnormal flavour in the lean ( $r=-0.11, P<0.05)$ or abnormal odour of the fat $(r=-0.09, \quad P<0.05)$ increased in larger pigs. Abnormal lean flavour and abnormal fat odour were highly correlated $(r=0.77, P<0.001)$. Abnormal flavour in the lean was most closely correlated with overall acceptability $(r=-0.73, P<0.001)$, followed by pork flavour in the lean $(r=0.60, P<0.001)$, abnormal fat odour $(r=-0.59, P<0.001)$, texture $(r=$ $0.44, P<0.001)$, pork odour of the fat $(r=0.35$, $P<0.001)$, juiciness $(r=0.32, P<0.001)$, shear force $(r$ $=-0.26, P<0.01)$ and cooked colour $(r=-0.17$, $P<0.01)$. Pork that had poorer waterholding capacity and was paler was less juicy based on correlations with drip loss

$$
(r=-0.26, P<0.01) \text { and lightness }(r=-0.13, P<0.01) \text {. }
$$

The conclusion from this work is that compared with gilts, boars produce carcasses which are leaner but of lower yield, and the meat, while it has slightly better water-holding capacity, has a lower overall eating quality. This is mainly because it has poorer pork flavour and is more likely to have abnormal flavour than meat from gilts. However, it is important to put these results into perspective. Instrumental measurements are objective ways of detecting differences in meat quality. Use of trained taste panels shows whether these differences are detectable by the human taster. Consumer panels are needed to refine this further. A difference detectable 
by a trained panel under controlled conditions of meat preparation and testing environment may be lost when meat is cooked by people in their homes. Only large-scale consumer testing could therefore tell us whether the differences between meat from boars and gilts found in the present work are commercially important. Nevertheless, any marketing schemes in which the eating quality of pork was pre-eminent should take the possibility of these effects being important into consideration.

\section{References}

Meat and Livestock Commission. 1989. Meat and Livestock Commission, Stotfold Pig Development Unit, First Trial. Milton Keynes, Meat and Livestock Commission.

Rhodes, D. N. 1972. Consumer testing of pork from boar and gilt pigs. Journal of the Science of Food and Agriculture 23: 1483-1491.

Warriss, P. D., Kestin, S. C., Rolph, T. P. and Brown, S. N. 1990. The effects of the beta-adrenergic agonist Salbutamol on meat quality in pigs. Journal of Animal Science 68:128-136. 